\title{
Overexpressed exocyst complex component 3-like 1 spontaneously induces apoptosis
}

\author{
Shunichi Matsumoto ${ }^{1 \#}$, Junichi Okada ${ }^{2 \#}$, Eijiro Yamada ${ }^{1}$, Tsugumichi Saito ${ }^{1}$, Kazuya Okada ${ }^{3}$, Takuya \\ Watanabe ${ }^{4}$, Yasuyo Nakajima ${ }^{1}$, Atsushi Ozawa ${ }^{1}$, Shuichi OKada ${ }^{1}$, and Masanobu Yamada ${ }^{1}$ \\ ${ }^{1}$ Department of Medicine and Molecular Science, Gunma University Graduate School of Medicine, 3-39-15 Showa-machi, Maebashi, \\ Gunma 371-8511, Japan; ${ }^{2}$ Department of Medicine, Division of Endocrinology, Albert Einstein College of Medicine, 1301 Morris Park \\ Ave., Price 369, Bronx, NY 10461, USA; ${ }^{3}$ Omagari Kousei Medical Center, 8-65 Omagari-torimachi, Daisen, Akita 014-0027, Japan; \\ and ${ }^{4}$ Department of Endocrinology and Metabolism, Saku Central Hospital Advanced Care Center, 28-3400 Nakagomi, Saku, Nagano \\ 385-0051, Japan
}

(Received 12 February 2021; and accepted 25 February 2021)

\begin{abstract}
Exocyst complex component 3-like 1 (EXOC3L1), which regulates insulin secretion, is ubiquitously present in heart, lung, liver, spleen, kidney, muscle, cerebellum, pituitary, adrenal grand, and pancreatic islets. Its deduced amino acid sequence has $31 \%$ identity and $53 \%$ similarity with Sec6, so they are considered isoforms. Since Sec6 suppresses apoptosis via HSP27, we investigated the involvement of EXOC3L1 expression in apoptosis. We found that overexpressed EXOC3L1 in Chinese hamster ovary cells significantly reduced cultured cell numbers. It also significantly increased apoptotic DNA ladder, caspase 3 activity, and cleavage of caspase 3 compared with the control. Thus, although Sec6 reduces apoptosis by increasing HSP27 phosphorylation, overexpressed EXOC3L1 alone can spontaneously induce apoptosis without apoptotic stimulators or inducers.
\end{abstract}

Exocytosis is an essential biological process in all eukaryotic cells. It functions in processes including intercellular communication and the release of materials and nutrients from cells, as well as mediating actions exerted on distantly located cells and tissues (Tanaka et al. 2017). Exocytosis is defined as the delivery of intracellular contents, including hormones, neural transmitters, mRNAs, miRNAs, and proteins, between intercellular membrane compartments and the plasma membrane; it is mediated by the soluble $\mathrm{N}$-ethylmaleimide-sensitive factor attachment protein receptor (SNARE) (Tanaka et al. 2017). Before secretory vesicles can fuse with the plasma membrane, they must be anchored to intercellular membrane

Address correspondence to: Shuichi Okada Department of Medicine and Molecular Science, Gunma University Graduate School of Medicine, 3-39-15 Showa-machi, Maebashi, Gunma 371-8511, Japan Tel: +81-27-220-8501, Fax: +81-27-220-8136

E-mail: okadash@gunma-u.ac.jp compartments by the exocyst complex (Tanaka et al. 2017). The exocyst complex is an evolutionarily conserved eight-protein complex, consisting of Sec3 (EXOC1), Sec5 (EXOC2), Sec6 (EXOC3), Sec8 (EXOC4), Sec10 (EXOC5), Sec15 (EXOC6), Exo70 (EXOC7), and Exo84 (EXOC8) subunits (Tanaka et al. 2017). The exocyst complex was originally known as the Sec6/8 complex. Rat Sec6 cDNA was predicted to encode an $87-\mathrm{kDa}$ protein with $22 \%$ amino acid identity to yeast Sec6p. Sec6 mRNA and Sec8 mRNA are present in the brain, spleen, lung, liver, muscle, kidney, and testis, with the highest expression in the brain, lung, muscle, kidney, and testis (Ting et al. 1995). Protein levels of Sec6 are also detected in the brain, lung, liver, kidney, testis, and pancreas (Kee et al. 1997). The Sec6 gene has a unique transcription initiation site. Primer extension analysis has revealed that the promoter is embedded in a $\mathrm{CpG}$ island and lacks the canonical TATA or

\footnotetext{
"These authors contributed equally to this work.
} 
CAAT boxes (Chin et al. 2000). These features are found in housekeeping gene promoters and neuronspecific gene promoters such as synapsins (Chin et al. 1994) and glutamate receptors (Myers et al. 1999).

Exocyst complex component 3-like 1 (EXOC3L1) was originally isolated and reported as an isoform of Sec6 to regulate insulin secretion in pancreatic $\beta$ cells (Saito et al. 2008). The mouse Exoc3L1 gene consists of 14 exons (coding exons from 2 to 14) and is located at chr8qD3. The Exoc3L1 gene is highly conserved in humans and rats. The deduced amino acid sequence of its product (EXOC3L1) has $31 \%$ identity and $53 \%$ similarity with that of Sec6. Like Sec6, EXOC3L1 is also ubiquitously expressed (Saito et al. 2008). Sec6 has been reported to suppress apoptosis by elevating the phosphorylation of HSP27 (Tanaka et al. 2008). We hypothesized that EXOC3L1 might affect apoptosis, like Sec6, because EXOC3L1 is an isoform of Sec6. We thus investigated that possibility in this study.

The reagents used in this study were as follows. The Apoptotic DNA Ladder Isolation Kit (ab65627) for cultured cells was purchased from Abcam (Cambridge, UK). The Live/Dead Cell Staining Kit II (PKCA707-3002) was procured from PromoCell (Heidelberg, Germany). Anti-caspase 3 (9662) and $\alpha$-tubulin (2144) antibodies were purchased from Cell Signaling Technology (Danvers, MA, USA). Horseradish peroxidase-conjugated anti-rabbit (31460) and -mouse (31437) IgG antibodies were acquired from Thermo Fisher Scientific (Waltham, MA, USA). All cell culture media and reagents were purchased from Thermo Fisher Scientific. All other chemicals used in this study were purchased from Sigma-Aldrich.

The materials used in this study were as follows. We used Chinese hamster ovary (CHO) cells (CRL12023) because our transfection efficiency was $\sim 100 \%$ (Okada et al. 1997). Cells were purchased from American Type Culture Collection and maintained in Minimum Essential Medium Eagle-alpha modified medium supplemented with $10 \%$ fetal bovine serum (Okada et al. 1997). To determine the function of EXOC3L1, it was transiently overexpressed by electroporation (Okada et al. 1997) using an expression vector purchased from OriGene (AK092858). The expression of EXOC3L1 was confirmed by western blotting using an EXOC3L1 antibody purchased from OriGene (TA324393). The apoptosis DNA ladder method was performed using the Apoptosis DNA Ladder Assay Kit, in accordance with the manufacturer's instructions. Cells were briefly lysed with Tris-EDTA lysis buffer and mixed with the enzyme solution supplied with the kit. After the isolation and purification of DNA, the samples were analyzed by agarose gel electrophoresis and ethidium bromide-stained DNA was visualized by ultraviolet transillumination. The activity of caspase 3 was estimated using the caspase 3 assay kit (CASP3-C; Sigma-Aldrich-Japan, Tokyo, Japan), in accordance with the manufacturer's instructions. Cells were briefly lysed with the supplied lysis buffer and mixed with the supplied caspase 3 substrate. After 90 min of incubation at $37^{\circ} \mathrm{C}$, the absorbance was read at $405 \mathrm{~nm}$. A positive control and a negative control were established, and caspase 3 activity was calculated in accordance with the manufacturer's instructions. The live cell assay was also performed in accordance with the manufacturer's instructions. Cells were initially grown in the wells of a 96-well microplate. After washing the cells in a serum-free buffer, $1 \mu \mathrm{M}$ calcein-AM was added to each well. The wells were then incubated at room temperature for 30-45 min and were finally analyzed by measuring the fluorescence using a microplate reader. All data in the figures are expressed as mean \pm standard deviation. Data were analyzed using one-way ANOVA to compare the means of all groups. The TukeyKramer multiple comparison procedure from the InStat 2.00 program was used to determine the statistical significance of differences between the means. A $p$ value of $<0.05$ was considered statistically significant.

As shown in Fig. 1a, the level of overexpressed EXOC3L1 protein was maintained for $24 \mathrm{~h}$ after electroporation, at which point it gradually declined. As previously reported, the transfection efficacy by our electroporation system for CHO cells was nearly 100\% (Okada et al. 1997). Therefore, the decrease in EXOC3L1 expression $48 \mathrm{~h}$ after electroporation means that those cells remaining on the culture dish after electroporation represent either electroporated cells that failed to take up the plasmid or those that took up a really small amount of plasmid. In addition, exogenously expressed EXOC3L1 might have a relatively short half-life. Therefore, to assess the new physiological function of EXOC3L1, we frozen the electroporated cells after $24 \mathrm{~h}$ and stored them at $-80^{\circ} \mathrm{C}$ until further use. As illustrated in Fig. $1 \mathrm{~b}$, the cell number remaining on the culture dish was reduced after EXOC3L1 overexpression. The proportion of round cells rather than spindle-shaped cells was increased by EXOC3L1 overexpression. The extent of the reduction in cell number from the culture dish was quantified and presented as a bar graph (Fig. 1c). When we estimated the number of 


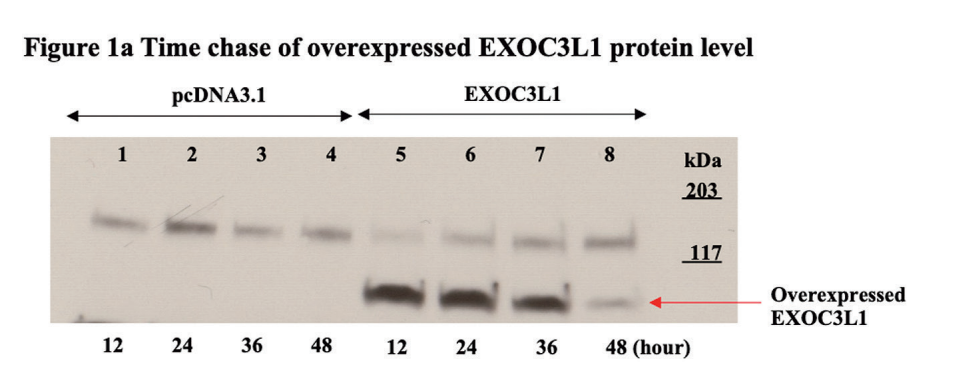

Figure 1b Microscopic observation
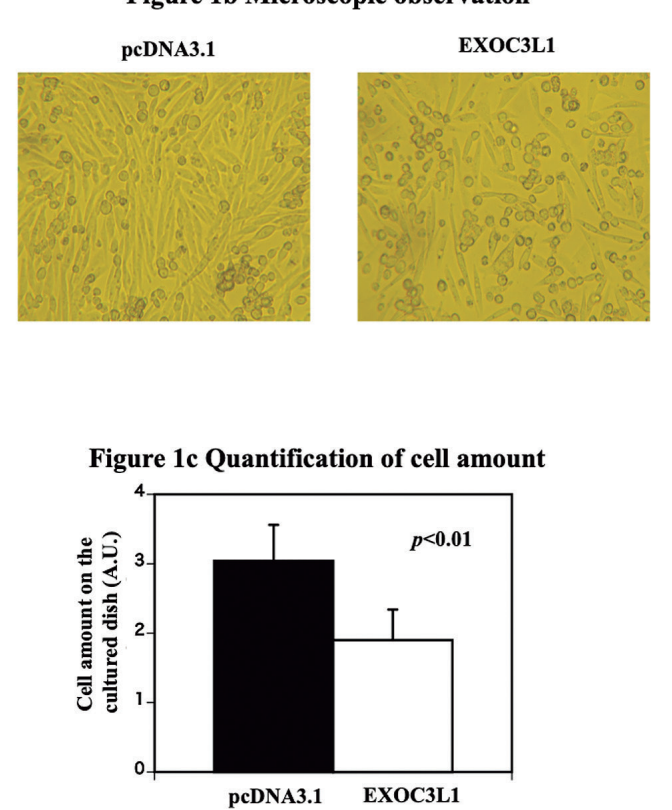

Fig. 1 Effect of overexpressed EXOC3L1 on cultured cells. a: EXOC3L1 was overexpressed by electroporation in CHO cells, as described in the text. Lanes 1-4 represent samples transfected with pcDNC3.1 vector. The exposure time was too short to visualize endogenous EXOC3L1 protein. Longer exposure times resulted in the burnout of overexpressed EXOC3L1 signals (data not shown). The numbers shown at the bottom of the picture represent the time after electroporation. Lanes 5-8 represent the overexpressed EXOC3L1 protein. The expression level of EXOC3L1 started to decline after $36 \mathrm{~h}$ following electroporation. The red arrow indicates EXOC3L1. The experiments were independently conducted in triplicate. b: Phase-contrast microscopy images are presented ( $\times 100$ magnification). The left panel shows cells transfected with an empty pcDNA3.1 vector, and the right panel shows cells with EXOC3L1 overexpression. The experiments were conducted independently in triplicate, and representative results are presented. c: Effects of overexpressed EXOC3L1 in $\mathrm{CHO}$ cells were quantified and presented as a bar graph. The y-axis represents the number of cells attached to the culture well as described in the text. The closed column represents cells transfected with an empty pcDNA3.1 vector, and the open column represents cells with EXOC3L1 overexpression. The experiments were conducted independently in triplicate. The overexpression of EXOC3L1 significantly increased caspase 3 activity $(P<0.01)$.

cells remaining on the culture dish by protein assay using whole-cell lysate, we obtained similar results, as shown in Fig. 1c. Thus, it became obvious that overexpressed EXOC3L1 reduced the cell number. These experiments were repeated three times.

To identify the mechanism involved in the reduction of cell numbers from the culture dish after EXOC3L1 overexpression, we determined the effects of overexpressed EXOC3L1 on apoptosis. As depicted in Fig. 2, EXOC3L1 overexpression increased the appearance of the apoptosis DNA ladder (Fig. 2a) concomitant with an increment of caspase 3 activity (Fig. 2b) and cleavage of caspase 3 (Fig. 2c). EXOC3L1 was originally isolated and reported as an isoform of Sec6 to regulate insulin secretion in pancreatic $\beta$ cells (Saito et al. 2008). The deduced amino acid sequence of EXOC3L1 has 31\% identity and 53\% similarity with that of Sec6. Like Sec6, EXOC3L1 is also ubiquitously expressed (Saito et al. 2008). It has been reported that Sec6 suppresses apoptosis (Tanaka et al. 2008). In this study, overexpressed EXOC3L1 significantly reduced cell numbers from the culture dish. We also found that it increased caspase 3 activity and apoptosis, as indicated by increases of apoptotic DNA ladder appearance and cleavage of caspase 3. Although EXOC3L1 resembles Sec6 in terms of the deduced amino acid sequence, we discovered that overexpressed EXOC3L1 did not suppress apoptosis but instead spontaneously induced it.

We confirmed that Sec5 is a binding partner of EXOC3L1 by designing a pull-down experiment, as previously reported (Saito et al. 2008) (data not shown). Therefore, we suggest that EXOC3L1 is not a simple isoform of Sec6. In fact, we did not observe enhanced HSP27 phosphorylation after EXOC3L1 overexpression, which differs from the effect of Sec6 (data not shown). At present, the mechanism through which overexpressed EXOC3L1 induces apoptosis is unknown. Interestingly, the Ser479 resi- 
Figure 2a DNA Ladder method

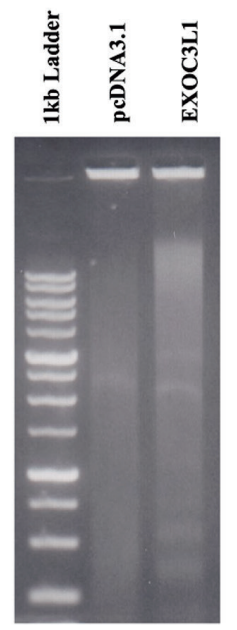

Figure 2c Caspase 3 cleavage

Figure 2b Caspase 3 activity

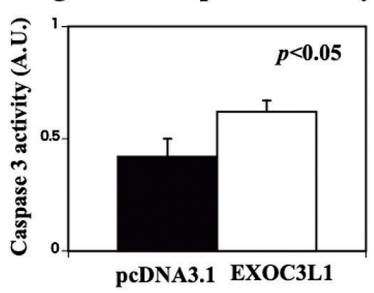

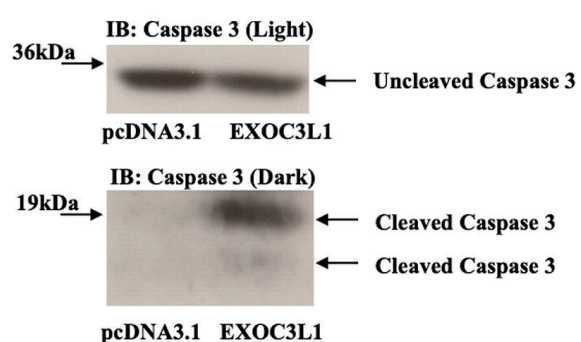

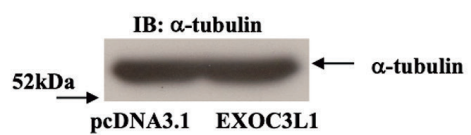

Fig. 2 Effect of overexpressed EXOC3L1 on apoptosis. a: Apoptotic DNA fragmentation was qualitatively analyzed using the DNA Ladder method. Lanes, from left to right: 1-kb DNA ladder marker; pcDNA3.1; and EXOC3L1. Analysis showed that overexpressed EXOC3L1 in cells caused apoptosis. Experiments were independently performed in triplicate and representative results are presented. b: Caspase 3 activity was quantitatively analyzed as described in the text. The closed column represents cells transfected with an empty pcDNA3.1 vector, and the open column represents cells with EXOC3L1 overexpression. The experiments were conducted independently in triplicate. The overexpression of EXOC3L1 significantly increased caspase 3 activity $(P<0.05)$. c: Under EXOC3L1 overexpression, CHO cells showed increased caspase 3 protein cleavage. Top panel: levels of uncleaved caspase 3 proteins (arrows) in cells with (right lane) and without EXOC3L1 overexpression (right lane). Middle panel: levels of cleaved caspase 3 proteins (arrows) in the presence (right lane) and absence of EXOC3L1 overexpression (right lane). Bottom panel: $a$-tubulin IB shows equivalent protein loading in each lane. Experiments were independently performed in triplicate.

due in EXOC3L1 is surrounded by a consensus motif of a protein kinase $\mathrm{C} \zeta(\mathrm{PKC} \zeta)$ phosphorylation site (Scansite $4.0 \mathrm{https}: / /$ scansite4.mit.edu/4.0/\#home), a component that does not exist in Sec6. PKC $\zeta$ has been reported to induce carcinogenesis by phosphorylating S71 in Rac1 (Islam et al. 2018). Therefore, it is possible that overexpressed S479 on EXOC3L1 and overexpressed S71 on Rac1 compete with each other as a substrate for $\mathrm{PKC} \zeta$. Further studies are required to clarify the underlying mechanisms.

In conclusion, the overexpression of EXOC3L1 in cells would enhance apoptosis even when endogenous EXOC3L1 expression is extremely limited. It would be interesting to determine how endogenous EXOC3L1 is involved in the regulation of insulin secretion.

\section{Acknowledgments}

There was no financial support for this study.

\section{CONFLICT OF INTEREST}

The authors declare no conflicts of interest.

\section{REFERENCES}

Chin LS, Li L and Greengard P (1994) Neuron-specific expression of the synapsin II gene is directed by a specific core promoter and upstream regulatory elements. $J$ Biol Chem 269, 18507-18513.

Chin LS, Weigel C and Li L (2000) Transcriptional regulation of gene expression of sec6, a component of mammalian exocyst complex at the synapse. Brain Res Mol Brain Res 79, 127-137.

Islam SMA, Patel R and Acevedo-Duncan M (2018) Protein kinase $\mathrm{C}-\zeta$ stimulates colorectal cancer cell carcinogenesis via PKC- $\zeta /$ Rac $1 /$ Pak1/ $\beta$-catenin signaling cascade. Biochim Biophys Acta Mol Cell Res 1865, 650-664.

Kee Y, Yoo JS, Hazuka CD, Peterson KE, Hsu SC, et al. (1997) Subunit structure of the mammalian exocyst complex. Proc Natl Acad Sci USA 94, 14438-14443.

Myers SJ, Dingledine R and Borges K (1999) Genetic regulation of glutamate receptor ion channels. Annu Rev Pharmacol Toxicol 39, 221-241.

Okada S, Kao AW, Ceresa BP, Blaikie P, Margolis B, et al. (1997) The $66-\mathrm{kDa}$ Shc isoform is a negative regulator of the epidermal growth factor-stimulated mitogen-activated protein kinase pathway. J Biol Chem 272, 28042-28049.

Saito T, Shibasaki T and Seino S (2008) Involvement of Exoc31, a protein structurally related to the exocyst subunit Sec6, in insulin secretion. Biomed Res (Tokyo) 29, 85-91.

Tanaka T, Goto K and Iino M (2017) Diverse functions and signal transduction of the exocyst complex in tumor cells. $J$ Cell Physiol 232, 939-957. 
Tanaka T, Iino M and Goto K (2018) Sec6 enhances cell migration and suppresses apoptosis by elevating the phosphorylation of p38 MAPK, MK2, and HSP27. Cell Signal 49, 1-16.

Ting AE, Hazuka CD, Hsu SC, Kirk MD, Bean AJ, et al. (1995)
rSec6 and rSec8, mammalian homologs of yeast proteins essential for secretion. Proc Natl Acad Sci USA 92, 96139617. 